\title{
Europäische Wissensordnung im Schweizer \\ Fremdsprachenunterricht \\ (1961-1990)
}

\section{Sandra Grizelj und Daniel Wrana}

Der Europarat ist seit seiner Gründung 1949 ein wichtiger Akteur auf dem Gebiet der Fremdsprachen-Policy. Mittels Resolutionen und Empfehlungen setzte er programmatische Ziele für den schulischen Fremdsprachenunterricht, die für die Mitgliedländer nicht verbindlich waren, aber dennoch über kulturelle Transfers Prozesse einer Neuordnung schulischen Wissens in Gang setzten. Die EDKnahm die Empfehlungen des Europarates auf und formulierte 1975 ein nationales Curriculum für Fremdsprachen. Damit sollten die Landessprachen gefördert und die europäische Kulturpolitik umgesetzt werden. Auf kantonaler Ebene verzögerte sich die Neuordnung des Wissens aus pragmatischen und schulorganisatorischen Gründen.

\section{Einleitung}

Bis in die 1990er Jahre war in fast allen Kantonen der Schweiz die erste in der Volksschule unterrichtete Fremdsprache eine Landessprache (Extermann, 2013; Stauffacher, 2016; Stauffer, 1999). In den Debatten zum schulischen Wissen wird daher der Unterricht in der ersten Fremdsprache meist als Unterricht in der zweiten Landessprache thematisiert. Lange war dieses Schulfach Schülerinnen und Schülern vorbehalten, die einen Schultyp mit erweiterten Leistungsansprüchen besuchten. Ab den 1960er und 70er Jahren begann die Einführung des Fremdsprachenunterrichts zumindest als Wahlfach auf allen Leistungsniveaus der Sekundarstufe I (EDK, 1974a; Egger \& Blanc, 1977; Giudici \& Grizelj, 2014, 2017).

Die Stellung der Landessprachen in der schulischen Wissensordnung wurde durch die Empfehlungen und Beschlüsse betreffend Einführung, Reform und Koordination des Unterrichts in der zweiten Landessprache für alle Schüler während der obligatorischen Schulzeit vom 30. Oktober 1975 der Schweizerischen Konferenz der kantonalen Erziehungsdirektoren (EDK) zusätzlich gestärkt. Darin war vorgeschrieben, dass die erste Fremdsprache nicht Englisch sein durfte, sondern eine der drei Landessprachen Deutsch, Französisch oder Italienisch zu sein hatte. Des Weiteren empfahl die EDK den Beginn des Unterrichts in der zweiten 
Landessprache im 4. oder 5. Schuljahr (EDK, 1975). Die Umsetzung dieser beiden Empfehlungen erfolgte in den meisten Kantonen bis spätestens Ende der 1990er Jahre. ${ }^{1}$

Ein Blick über die Landesgrenze zeigt, dass sowohl die Einführung des Fremdsprachenunterrichts auf allen Leistungsniveaus der Sekundarstufe I als auch seine Vorverlegung auf die Primarstufe in anderen europäischen Ländern in einem ähnlichen Zeitraum begann wie in der mehrsprachigen Schweiz. Nach dem Zweiten Weltkrieg wurde das Fremdsprachenfach allmählich auf allen Leistungsniveaus der Sekundarstufe I eingeführt (Christ \& de Cillia, 2003). Spätestens in den 1990er Jahren setzte sich die Praxis des frühen Fremdsprachenunterrichts in den europäischen Ländern durch (H. Christ, 2003; I. Christ, 2003).

In den Empfehlungen von 1975 legitimierte die EDK ihre Bemühungen in den «einleitenden Erwägungen» (EDK, 1975, S. 23) nicht nur mit dem Argument der mehrsprachigen Schweiz, sondern verwies explizit auf den europäischen Kontext. Mit den Empfehlungen trüge die Schweiz «zur Verwirklichung einer europäischen Kulturpolitik bei, die schon im Jahre 1961 durch die Erziehungsminister der Europaratländer ins Auge gefasst wurde» (EDK, 1975, S. 23). Ausserdem hätte sich in den 1960er Jahren «in mehreren europäischen Ländern eine Entwicklung abgezeichnet, die eine wesentliche Erweiterung der fremdsprachlichen Kenntnisse und Fertigkeiten aller Schüler durch Vorverlegung des Fremdsprachunterrichts und durch betonte Pflege des mündlichen Ausdrucks als möglich erscheinen liess» (EDK, 1975, S. 23). Das «Schulfach Fremdsprache» wurde dies- und jenseits der Landesgrenze Teil schulischen Wissens in allen Leistungsniveaus der Sekundarstufe I sowie auf der Primarstufe.

Die historische Entwicklung der Schulfächer insgesamt - und damit diejenige des Schulfaches Fremdsprache - zeigt, dass die schulische Wissensordnung ein Produkt gesellschaftlicher Konstruktionsprozesse ist. Das schulische Wissen ist weit davon entfernt, eine blosse Reduktion und Rekonstruktion wissenschaftlichen Wissens zu sein (Goodson 1997, 1999). Ebenso entscheidend wie der Bezug zur wissenschaftlichen Disziplin ist der gesellschaftliche Bezug. Wissen ist "all das Wissen, was als solches gilt, also als solches durch eine bestimmte gesellschaftliche Instanz legitimiert ist» (Namer, 1981, S. 192; Hervorh. im Original). In Bezug auf die Schule heisst das, dass schulisches Wissen dasjenige Wissen ist, das durch politisch legitimierte Instanzen in bestimmten Verfahren als solches legitimiert wird. Es wird permanent revidiert und von verschiedenen gesellschaftlichen Akteuren immer wieder neu verhandelt. Dies gilt auch und gerade für den Unterricht in der zweiten Landessprache bzw. den Fremdsprachenunterricht im Allgemeinen. Die expliziten Verweise in den Empfehlungen der EDK von 1975 sowohl auf die Mehrsprachigkeit der Schweiz als auch auf die Bemühungen der Europaratländer - und damit des Europarats - werfen zwei Fragen auf: Wurde anhand von kulturellen Transfers die Erweiterung des Fremdsprachenunterrichts in der Schweiz durch diejenige im Europarat «angestossen»? Wie hat sich die schulische Wissensordnung hinsichtlich des Schulfaches Fremdsprache in 
der Folge der Empfehlungen der EDK verändert und wie wurde dies kantonal legitimiert? Diese Fragen werden in vorliegendem Artikel diskutiert und analysiert.

Die Untersuchung fokussiert auf die Beziehung bzw. die kulturellen Transfers im Hinblick auf den Fremdsprachenunterricht zwischen der EDK und dem Europarat. ${ }^{2}$ Der Untersuchungszeitraum beginnt 1961. In diesem Jahr verabschiedete der Europarat die erste wichtige Resolution zum Fremdsprachenunterricht (Trim, 2007). Für die erste Frage nach den kulturellen Transfers wird die Zeit bis 1975 untersucht, weil in diesem Jahr die EDK die Empfehlungen veröffentlichte. Für die Frage nach der Veränderung der schulischen Wissensordnung und der kantonalen Legitimation wird der Untersuchungszeitraum bis 1990 ausgedehnt. Der Fokus richtet sich auf vier Kantone in der deutschsprachigen Schweiz: Aargau, Bern, Schwyz und Zürich.

Als Quellenmaterial dienen die verschiedenen Empfehlungen und Regelungen des Europarates in Bezug auf den Unterricht von Fremdsprachen. Für die Schweiz werden Jahresberichte der EDK (für die Jahre von 1968 bis 1976) ${ }^{3}$, verschiedene Akten der EDK sowie ihre Informationsbulletins zum Fremdsprachenunterricht untersucht. ${ }^{4}$ Das Quellenmaterial der Kantone setzt sich aus Lehrplänen, Gesetzen und Rechenschaftsberichten zusammen. Des Weiteren werden Artikel zum Thema aus dem «Archiv für das schweizerische Unterrichtswesen», aus der «Schweizerischen Lehrerzeitung» und der «Schweizer Schule» beigezogen.

In der Forschung zum Fremdsprachenunterricht dominieren vor allem Untersuchungen zu didaktischen, methodischen, kommunikations- oder kompetenzorientierten Aspekten, die keine historische Perspektive einnehmen (z.B. Haas, 2010; Hutterli, 2011; Lüdi, Pekarek \& Saudan, 2001; Peyer, Andexlinger, Kofler \& Lenz, 2016). Insbesondere der frühe Fremdsprachenunterricht auf der Primarstufe wird oft nach seiner Wirksamkeit evaluiert (z.B. für die deutschsprachige Schweiz: Bader \& Schaer, 2005; Husfeldt \& Bader Lehmann, 2009; Haenni Hoti, Müller, Heinzmann, Wicki \& Werlen, 2009). Sprachenpolitik als Forschungsfeld ist bisher nicht systematisch strukturiert (Krumm, 2016a). Der europäische Bezug geschieht vor allem im Hinblick auf die Standortbestimmung nach dem Gemeinsamen Europäischen Referenzrahmen (GER) (z.B. Schwob \& Hexel, 2008). ${ }^{5}$ Bislang fehlen historische Untersuchungen zu kulturellen Transfers im Bereich des Fremdsprachenunterrichts zwischen der Schweiz und dem Europarat.

Als theoretische Rahmung für die Untersuchung der Forschungsfragen dient der Ansatz des Kulturtransfers. ${ }^{6}$ Die übergreifende These des Kulturtransfers besagt, dass lokale, nationale und transnationale Prozesse ständig ineinandergreifen. Dadurch wird pädagogisches Wissen wechselseitigen Veränderungen, Bedeutungsverschiebungen und Reinterpretationen unterworfen (Caruso, Koinzer, Mayer \& Priem, 2014; Fontaine, 2015). In vorliegender Untersuchung stehen die transnationalen Transfers und Grenzüberschreitungen im Bildungsbe- 
reich mit Fokus auf das Schulfach Fremdsprache im Mittelpunkt. Hierbei werden die Produktion, der Transfer und die Rezeption von Wissen ins Zentrum gerückt. Der Kulturtransferansatz zielt darauf ab, das national verankerte Verständnis der historischen Bildungsforschung aufzubrechen (Caruso et al., 2014).

In einem ersten Schritt werden die verschiedenen Förderprogramme bzw. die wichtigsten Empfehlungen oder Resolutionen für den Fremdsprachenunterricht des Europarates vorgestellt. Anschliessend wird gezeigt, in welchem Kontext die Empfehlungen der EDK entstanden sind und welche Ziele sie verfolgten. Im folgenden Kapitel soll die Frage geklärt werden, welche kulturellen Transfers zwischen dem Europarat und der Schweiz die Erweiterung des Fremdsprachenunterrichts in der Schweiz anstiessen. Anschliessend wird gezeigt, wie sich die schulische Wissensordnung in den untersuchten Kantonen verändert hat und wie dies legitimiert wurde. Abschliessend werden die beiden Fragen nach den kulturellen Transfers und der Wissensordnung zueinander in Beziehung gebracht und gemeinsam diskutiert.

\section{Die Förderung des Fremdsprachenunterrichts durch den Europarat}

Der Europarat (Council of Europe, Conseil de l'Europe [CE]) wurde 1949 gegründet. ${ }^{7}$ Es handelt sich um eine zwischenstaatliche Organisation, der heute 47 Staaten angehören und der die Schweiz am 6. Mai 1963 als 17. Mitglied beitrat. In den 1950er Jahren sah man in der Schweiz von einem Beitritt ab aus Sorge um die Neutralität. Wirtschaftspolitische Interessen der exportorientierten Schweiz waren schliesslich ein wichtiger Grund für den Beitritt (Wyder, 1984).

Schon bald nach seiner Gründung befasste sich der Europarat intensiv mit dem Fremdsprachenunterricht. Gemäss Artikel 2 des Europäischen Kulturabkommens vom 19. Dezember 1954 - das die Schweiz noch vor dem Betritt zum Europarat am 13. Juli $1962^{8}$ unterzeichnete - verpflichteten sich die ratifizierenden Staaten u.a. dazu, «das Studium der Sprachen, der Geschichte und der Zivilisation der anderen Vertragsparteien» zu fördern (CE, 1954 [2003] Art. 2 lit. a). Das Kulturabkommen war v.a. als «Manifest der Zusammengehörigkeit konzipiert» (Wyder, 1984, S. 374).

Um die im Kulturabkommen verankerten sprachenpolitischen Ziele und Projekte umsetzen zu können, wurde 1957 eine sprachenpolitische Abteilung (language policy divison; heute language policy unit) eingerichtet (Krumm, 2016b). Die Abteilung entwickelte Instrumente und Materialien, um das kommunikative und kompetenzorientierte Sprachenlernen zu fördern (Trim, 2007). Der Europarat verfolgte damit das übergeordnete Ziel, die Quantität und Qualität der Kommunikation zwischen den Einwohnerinnen und Einwohnern der Mitgliedstaaten zu fördern. Fremdsprachenunterricht «für alle» sollte ein bildungspolitisches Prinzip werden, und nicht mehr wie bis dahin in 
vielen europäischen Ländern als ein Privileg einer kulturellen oder gebildeten Oberschicht gelten (Stoks, 1994).

Anlässlich zweier Konferenzen in den Jahren 1961 und 1962 empfahlen die europäischen Erziehungsminister und -ministerinnen die Ausdehnung des Unterrichts in einer zweiten Sprache auf alle Schülerinnen und Schüler, unabhängig davon, welches Leistungsniveau sie besuchten (EDK, 1974b). Die Erziehungsminister forderten den Miteinbezug von Expertinnen und Experten bei der Ausbreitung des Fremdsprachenunterrichts: Jedes Land sollte Forschung im linguistischen und psychologischen Bereich unterstützen. Die Spezialisten sollten sich unter der Federführung des Europarats treffen, um methodische und andere Probleme des Unterrichts von modernen Sprachen zu diskutieren. Das Ministerkomitee folgte diesen Forderungen und verabschiedete 1961 die erste grosse und wichtige Resolution bezüglich des Fremdsprachenunterrichts (Trim, 2007).

Acht Jahre später folgte 1969 eine weitere wichtige Resolution, deren Titel Programm war und die Bedeutung des Fremdspracheunterricht für Europa unterstrich: «Programme intensifié d'enseignement des langues vivantes pour l'Europe» (CE, 1969). Verschiedene programmatische Feststellungen leiteten die eigentlichen Empfehlungen ein und dienten der Legitimation einer europäischen Sprachenpolitik. Die sprachliche Vielfalt wurde als Teil des kulturellen europäischen Erbes und Bereicherung verstanden und nicht als ein Hindernis zur Einheit. Nur eine Verallgemeinerung des Studiums der europäischen Sprachen könne das Verständnis und die Zusammenarbeit in Europa ermöglichen. Kenntnisse der europäischen Sprachen seien kein Luxus für eine Elite, sondern ein Instrument für Information und Kultur, das jede und jeder besitzen müsse. Der Beginn des Fremdsprachenunterrichts von mindestens einer weit verbreiteten europäischen Sprache wurde ab einem Alter von ungefähr 10 Jahren empfohlen (CE, 1969).

Den Mitgliedstaaten wurde empfohlen, spezialisierte Zentren zu eröffnen, um Lehrpersonen und andere interessierte Personen über Forschungsergebnisse zum Unterricht in modernen Fremdsprachen zu informieren. Zudem sollten die Mitgliedstaaten «einen «Korrespondenten für moderne Sprachen» («un (correspondant de langues vivantes»") (CE, 1969, S. 10) nominieren, der mit der Aufgabe vertraut werden sollte, die Ziele des Rats für kulturelle Zusammenarbeit zu fördern und das europäische Programm, moderne Sprachen zu stärken, umzusetzen (CE, 1969). ${ }^{9}$ Ziel der Programme des Europarates war es, die verschiedenen Akteure im Feld des Fremdsprachenunterrichts zu vernetzen (Trim, 2007).

Es blieb jedoch nicht nur bei Resolutionen oder Empfehlungen auf Papier und Forderungen nach vermehrter Forschung. Der Rat für kulturelle Zusammenarbeit veranstaltete bis 1974 über 20 Symposien, an denen Bedeutung, Bedürfnisse und Beginn des Fremdsprachenunterrichts diskutiert wurden. Lernziele, Methoden für die verschiedenen Schultypen und Altersstufen, die 
Ausbildung von Lehrpersonen gehörten ebenso zu den Diskussionspunkten wie die Frage nach dem Forschungsbedarf (EDK, 1974b). Weitere Schwerpunkte der Forschung bezogen sich auf linguistische und psychologische Faktoren des Fremdsprachenerwerbs. Ein wichtiges Anliegen des Europarates war es, dass die Ergebnisse zwischen den Mitgliedstaaten ausgetauscht werden. Die Programme sollten einer Vernetzung der verschiedenen Akteure im Feld des Fremdsprachenunterrichts dienen (Trim, 2007).

Die Bemühungen um bessere Verständigung unter den Menschen sind klar im Kontext der Folgen des Zweiten Weltkriegs zu sehen. Um eine Einheit werden zu können, sollten die sprachlichen Barrieren innerhalb Europas beseitigt werden (CE, 1969). Das Erlernen von anderen Sprachen sollte die Menschen zusammenführen. Die EDK formulierte ähnliche Ziele in den Empfehlungen von 1975 mit Blick auf die Schweiz und Europa, wie das folgende Kapitel zeigt.

\section{Die Empfehlungen der EDK für den Fremdsprachenunterricht}

Die Entstehung der Empfehlungen der EDK für den Unterricht in der zweiten Landessprache sind im Rahmen des Beginns einer stärkeren Koordination der kantonalen Schulsysteme zu verorten. Ende der 1960er Jahre begannen erste Bemühungen der EDK, die kantonalen Schulsysteme zu koordinieren: der Schuljahresbeginn, das Schuleintrittsalter oder die Dauer der Schulpflicht sollten vereinheitlicht werden. Mit dem Schulkonkordat von 1970 erhielten diese Bestrebungen eine rechtliche Grundlage für die Zusammenarbeit zwischen den Kantonen (Manz, 2011). Neben dieser äusseren, organisatorischen Koordination wurde auch eine innere angestrebt, wie beispielsweise die Angleichung der Lehrpläne und Lehrmittel oder die Erneuerung des Mathematikunterrichts. Ein wichtiger Bereich der inneren Koordination betraf den Fremdsprachenunterricht (Badertscher, 1997ba).

Wie einleitend erwähnt, legitimierte die EDK ihre Empfehlungen zum Fremdsprachenunterricht von 1975 einerseits mit der Mehrsprachigkeit der Schweiz und andererseits mit den Bemühungen der Europaratländer. In Bezug auf die Schweiz ging es nicht nur darum, dass «Kenntnisse in einer zweiten Landessprache [...] für alle Schweizer von grossem staats- und kulturpolitischem Interesse [sind]» (EDK, 1975, S. 23). Ebenso sollte mit einem einheitlichen Beginn des Unterrichts der zunehmenden Mobilität der Bevölkerung zwischen den Kantonen Rechnung getragen werden und dadurch der Schulübertritt von einem Kanton in den anderen erleichtert werden. Mit der Vorverlegung «sollte eine Harmonisierung des Beginns des Unterrichts in der zweiten Landessprache angestrebt werden» (EDK, 1975, S. 23). Die EDK empfahl den früheren Beginn (im 4. oder 5. Schuljahr). Der Fremdsprachenunterricht sollte zudem didaktisch grundlegend reformiert und die Priorität neu auf mündlicher Kommunikation 
und Hörverstehen gelegt werden. Der «Erfahrungshorizont» (EDK, 1975, S. 29) der Schülerinnen und Schüler sollte erweitert werden, aber nicht nur im Hinblick auf die betreffende Sprachregion, sondern ebenso auf das entsprechende ausländische Sprachgebiet.

Die in den Empfehlungen der EDK formulierten Ziele wurden allerdings nicht neu «erfunden", sondern über Prozesse kultureller Transfers vom Europarat übernommen und an Schweizer Verhältnisse angepasst.

\section{Transnationale kulturelle Transfers beim Fremdsprachenunterricht}

Pädagogische Ideen und Konzepte verbreiten sich nicht aus eigenem Antrieb. Sie benötigen verschiedene Vermittlungsinstanzen (Caruso et al., 2014, S. 42). Kulturelle Transfers in Bezug auf den Fremdsprachenunterricht verliefen zwischen dem Europarat und der Schweiz über verschiedene Vermittlungsinstanzen: über Kommissionen und Delegierte, die an Symposien und Erziehungsministerkonferenzen teilnahmen, über Lehrmittel oder über den Austausch von Forschungsergebnissen. Ideen und Praktiken konnten sich so verbreiten. Der EDK kam im Transferprozess des Fremdsprachenunterrichts, bei dem transnationale und nationale Prozesse ineinandergriffen, eine zentrale Stellung zu. Die folgenden Abschnitte zeigen, welche Vermittlungsinstanzen zwischen dem Europarat und der EDK den kulturellen Transfer antrieben.

Der Betritt der Schweiz zum Europarat im Jahr 1963 stellte die EDK vor neue Aufgaben. Sie war einerseits Vertreterin der Kantone innerhalb der Schweiz und andererseits vertrat sie die Schweiz in der europäischen Zusammenarbeit. Aufgrund der föderalistischen Struktur der Schweiz fällt die Bildungspolitik in den Aufgabenbereich der Kantone. Für den Europarat ist die föderalistische Struktur ebenso kennzeichnend wie für die Schweiz (Wyder, 1984). ${ }^{10}$ Um die Schweiz im Europarat vertreten zu können, stimmte die EDK der Schaffung einer ständigen Kommission für internationale Zusammenarbeit zu. Die Wahrnehmung solcher Aufgaben war für die EDK wichtig:

Die Erziehungsdirektorenkonferenz muss mitreden bei der Bestellung der Delegationen, sie muss diesen ihre Instruktionen und allenfalls Kompetenzen erteilen. Sie soll aber auch besorgt sein darum, dass die an diesen Fragen interessierten Kreise in der Schweiz über die europäischen Erfahrungen und Bestrebungen informiert werden und dass vor allem in unserem Lande die Tätigkeiten, die aus diesen internationalen Kontakten erwachsen (Europarat, OCDE, Unesco, BIE usw.) richtig koordiniert werden («Die Tagungen der Erziehungsdirektorenkonferenz 1963", 1964, S. 111, Hervorh. im Original).

Der EDK wurde ein kompetenter und zentraler Akteur bezüglich der internationalen Kontakte. Gleichzeitig war sie eine Vermittlerin, indem sie die Informationen zu den europäischen Erfahrungen und Bestrebungen innerhalb der 
Schweiz weitergab. ${ }^{11}$ Die Erziehungsministerkonferenzen des Europarates boten ihr die Möglichkeit für Vernetzung - nicht nur in Bezug auf Fremdsprachenunterricht, sondern ebenso in anderen Bereichen des Bildungswesens. ${ }^{12}$

Der Bezug zum und die Orientierung am Europarat waren für die Fremdsprachen-Policy der EDK zentral. Der Europarat war und ist ein wichtiger Akteur in diesem Gebiet. Von Anfang an beschäftigten Sprache und Bildung die europäischen Erziehungsminister. Viele der vom Europarat verabschiedeten Empfehlungen oder Resolutionen bildeten eine Grundlage für gemeinsame Sprachbildungspolitiken in Europa und sollten der Entwicklung eines Europa dienen, das durch seine mehrsprachigen Bürger und Bürgerinnen charakterisiert ist (Beacco \& Byram, 2007).

Die Arbeiten des Europarates stehen für Prozesse von Globalisierung und die Förderung der Mehrsprachigkeit (Sarter, 2013). Im Fremdsprachenunterricht manifestierten sich diese Prozesse, indem sich die Sprachenpolitiken der europäischen Länder mittels kultureller Transfers anglichen. Diese Angleichungen waren keine Top-down-Prozesse, sondern vielmehr wechselseitige Prozesse, in welche die Mitgliedstaaten von Anfang an eingebunden waren. Als Grundlage für die Bildung einer gemeinsamen Sprachen-Policy diente eine Bestandesaufnahme, die von der Erziehungsministerkonferenz in Auftrag gegeben wurde. Mithilfe einer vergleichenden Studie sollten die Strukturen und der Umfang des Fremdsprachenunterrichts in den Mitgliedstaaten eruiert werden. Die Umfrage, an der die Schweiz ebenfalls teilnahm, wurde in den Jahren 1962 und 1963 durchgeführt (Staatsarchiv Luzern, EDK, 1963).

Der Beitritt der Schweiz zum Europarat führte innerhalb der EDK zur Gründung von neuen Funktionsstellen und Kommissionen, die sich explizit mit dem Fremdsprachenunterricht befassten. Damit leistete die EDK den Forderungen des Europarates Folge. Im Jahr 1971 ernannte die EDK eine Projektleiterin sowie eine Kommission für das Projekt Fremdsprachenunterricht (EDK, 1972). Nur ein Jahr später wurde sie zur Expertenkommission zur Einführung und Koordination des Fremdsprachunterrichts in der obligatorischen Schulzeit umbenannt und mit einem Wissenschaftlichen Sekretariat für Fremdsprachenunterricht ausgestattet (EDK, 1973). Die Projektleiterin nahm eine Doppelfunktion ein, indem sie einerseits das Projekt Fremdsprachenunterricht in der Schweiz koordinierte. Andererseits fungierte sie als "Vertreterin des Bundes» (EDK, 1973, S. 12) bei Symposien und weiteren Gremien des Europarates. Aufgrund dieser Doppelfunktion war es ihr möglich, den Erziehungsdirektoren, der Expertenkommission der EDK, den Versuchsleitern (von Versuchen mit Französisch in der Primarschule) und den Lehrerseminaren Informationen aus dem Europarat zur Fremdsprachenfrage zu übermitteln (EDK, 1974c).

Die kulturellen Transferprozesse verliefen nicht nur formell über Gremien und Tagungen, sondern auch über Lehrmittel. Im Jahr 1969 starteten beispielsweise wissenschaftlich begleitete Versuche in den Kantonen Aargau, Bern und Zürich mit den Lehrmitteln «Frère Jacques» oder «Bonjour Line» (EDK, 1974a, 
1974b; Stillhart, 2005). Letzteres wurde auch in Versuchen in anderen Mitgliedstaaten des Europarates benutzt (Trim, 2007).

Ein weiteres wichtiges Feld für kulturelle Transfers war die Forschung, die hauptsächlich auf Untersuchungen zum frühen Fremdsprachenunterricht fokussierte. Ein Symposium des Europarats, an dem die Schweiz mit zwei Delegierten teilnahm, widmete sich diesem Thema im Jahr 1971. Es ging um die Frage, ob ein früherer Beginn zu besseren Kenntnissen der Fremdsprache führe bzw. welches aus psychologischer und pädagogischer Sicht das optimale Alter für den Beginn des Fremdsprachenunterrichts sei. In diesem Zusammenhang wurde u.a. auf eine Schweizer Studie (Bühler, 1972) verwiesen, die zeigte, dass die älteren Schülerinnen und Schüler bessere Resultate nach eineinhalb Jahren erzielten als solche, die im 4. oder 5. Schuljahr mit Französischunterricht begonnen hatten. Untersuchungen in anderen Ländern widersprachen diesen Ergebnissen. Trotz der unklaren Forschungslage wurde im Bericht ein früher Beginn empfohlen. Dies wurde mit pädagogischen und psychologischen Argumenten begründet (CE, 1974). Hier zeigen sich weitere Dimensionen der Legitimation für den Fremdsprachenunterricht. Es ging nicht nur um bessere Kommunikation zwischen den Mitgliedstaaten und den Zugang für alle zum Unterricht, sondern ebenso um pädagogische und psychologische Argumente. Auch diese finden sich in den Legitimationslinien der EDK wieder.

Der Expertenbericht, den die EDK in die Vernehmlassung schickte, führte in Bezug auf den früheren Beginn des Fremdsprachenunterrichts neben kulturpolitischen Argumenten physiologische, entwicklungspsychologische, erzieherische sowie unterrichtliche Argumente auf (EDK, 1974b). Die übergeordneten oder «ideellen» Ziele des Fremdsprachenunterrichts wie die verbesserte Kommunikation zwischen den Ländern, reichte nicht als Legitimation für einen frühen Beginn.

Diese Ausführungen zeigen, dass die Erweiterung des Fremdsprachenunterrichts durch kulturelle Transfers aus dem Europarat «angestossen» und über wechselseitige Beeinflussung zwischen den Europaratsländern weiter vorangetrieben wurde. Mit den Empfehlungen definierten sowohl der Europarat als auch die EDK programmatische Ziele, die für die Mitgliedländer bzw. die Kantone zwar nicht verbindlich waren, die aber dennoch Prozesse einer Neuordnung schulischen Wissens in Gang setzten. Im folgenden Kapitel wird untersucht, inwiefern sich die schulische Wissensordnung aufgrund der Empfehlungen der EDK von 1975 verändert hat. 


\section{Innernationale Transfers und Neuordnung des Wissens}

Die Koordination der Umsetzung der Empfehlungen übertrug die EDK den vier Regionalkonferenzen der EDK (EDK, 1975). ${ }^{13}$ Obwohl die EDK die Umsetzung den Regionalkonferenzen übertrug, war der Zeitpunkt der Einführung des frühen Fremdsprachenunterrichts von Kanton zu Kanton verschieden. Ein Vergleich der Lehrpläne der vier untersuchten Kantone zeigt, dass das schulische Wissen der Primarschule in allen Kantonen früher oder später neu geordnet wurde, doch in unterschiedlichem Tempo.

Die Kantone Aargau und Bern, beide Mitglieder der Regionalkonferenz der Nordwestschweiz (NW-EDK), zeigen die grösstmögliche zeitliche Spannweite. Im zweisprachigen Kanton Bern war der Französischunterricht bereits seit $1839 \mathrm{ab}$ dem 5. Schuljahr obligatorisch, allerdings nur in Sekundarschulen, dem Schultyp mit erweiterten Leistungsansprüchen ("Gesetz über die Sekundarschulen des Kantons Bern vom 12. März 1839»). ${ }^{14}$ Die Ausweitung des Obligatoriums für das 5. und 6. Schuljahr der Primaroberschule, dem Schultyp mit Grundansprüchen, erfolgte mit dem Lehrplan von 1983 (Erziehungsdirektion des Kantons Bern, 1983). Der Lehrplan von 1966 erlaubte Gemeinden, das Fach fakultativ einzuführen (Erziehungsdirektion des Kantons Bern, 1966). Im Kanton Aargau wurde der frühe Französischunterricht bis zum Ende des untersuchten Zeitraums (1990) nicht eingeführt, obwohl die NW-EDK 1977 empfahl, die Vorverlegung voranzutreiben («Kanton Aargau: Kontroverse um Französischunterricht», 1977). ${ }^{15}$

Auch andere Kantone führten den frühen Französischunterricht nach Ende des Untersuchungszeitraums ein. Die Regionalkonferenz der Innerschweiz (IEDK) sprach sich ebenfalls bald nach den Empfehlungen für die Vorverlegung aus und hielt auch daran fest (IEDK, 1985). Der Kanton Schwyz, Mitgliedkanton der IEDK, begann mit der Einführung des frühen Französischunterrichts im Jahr 1993 (Rechenschaftsbericht des Regierungsrates an den Kantonsrat, 1993). Die EDK-Ost empfahl den ihr angeschlossenen Kantonen die Vorverlegung des Französischunterrichts spätestens im Jahr 1996 ( Die Vorverlegung des Französischunterrichts in der Ostschweiz», 1986). Der Kanton Zürich, Mitglied der EDK-Ost, folgte dieser Empfehlung und führte das Fach mit dem Lehrplan von 1993 ein (Erziehungsdirektion des Kantons Zürich, 1993).

Beim innernationalen Transfer auf die kantonale Ebene ist eine grundlegende Bedeutungsverschiebung im Fremdsprachenunterricht festzustellen. Die «ideelle» Zielsetzung der verbesserten Kommunikationsfähigkeit innerhalb der Schweiz und den europäischen Ländern wich pragmatischen und schulorganisatorischen Legitimationen für die Nicht-Einführung bzw. verspätete Einführung des frühen Fremdsprachenunterrichts. Als Ausnahme bei den vier Kantonen ist der Kanton Bern zu sehen, der die Empfehlungen als erster umsetzte. Das lag insbesondere daran, dass Französisch die zweite Kantonssprache ist. 
Im Kanton Aargau äusserten sich in der Vernehmlassung sowohl der Erziehungsrat als auch die Lehrpersonen gegen eine Vorverlegung des Französischunterrichts. Die Ablehnung richtete sich vor allem gegen das vorgesehene Konzept der Einführung, das die Ausbildung der Mittelstufenlehrpersonen, die Ausarbeitung eines geeigneten Lehrmittels und die Weiterführung an der Oberstufe umfasste ("AG: Kontroverse um Französischunterricht», 1977). Aufgrund eines neuen Schulgesetzes im Jahr 1981 mussten die Lehrpläne revidiert werden. Der Erziehungsrat erteilte den Auftrag, für die 4. und 5. Primarschulklasse eine Stundentafel mit und ohne Französischunterricht zu erstellen, die anschliessend in die Vernehmlassung geschickt wurde. Die Stufenkommission Primarschule lehnte die Vorverlegung des Französischunterrichts grundsätzlich ab. Sie befürchtete u.a. einen Abbau an Stunden in Fächern der elementaren Kulturtechniken wie Lesen, Schreiben, Rechnen sowie Sach- und Heimatkunde. Die muttersprachlichen Fähigkeiten dürften nicht beeinträchtigt werden; ein früherer Beginn bedeute nicht einen effizienteren Unterricht und der zeitliche Aufwand seit zu gross für das, was erreicht werde (Erziehungsdepartement des Kantons Aargau, 1983).

Der Kanton Schwyz verzichtete zunächst auf eine Einführung aus rein pragmatischen Gründen. Seit Ende der 1970er Jahre lief ein anderes Reformprojekt, nämlich die Vorarbeiten für ein neues Mathematiklehrmittel von der 1. bis zur 9. Klasse: «Da nicht gleichzeitig zwei Reformprojekte in der Primarschule verwirklicht werden können, muss die allfällige Einführung des Französischunterrichts in der Primarschule auf das Ende der 80er oder Anfang der 90er Jahre verschoben werden.» (Rechenschaftsbericht des Regierungsrates an den Kantonsrat, 1980, S. 54). Am 31. Mai 1989 fällte der Schwyzer Erziehungsrat den Entscheid, Französisch in der Primarschule einzuführen (Rechenschaftsbericht des Regierungsrates an den Kantonsrat, 1989). Das Einführungskonzept sah eine schrittweise Einführung vor nach Bezirken und mit Beginn ab 1993 (Rechenschaftsbericht des Regierungsrates an den Kantonsrat, 1990). Vorher wurde jedoch die entsprechende Ausbildung an den Seminaren in die Wege geleitet (Rechenschaftsbericht des Regierungsrates an den Kantonsrat, 1988).

In der Ostschweiz (und damit im Kanton Zürich) waren die Lehrpersonen gegenüber der Einführung von frühem Französischunterricht sehr kritisch eingestellt. Im Jahr 1979 hält die Ostschweizer Regionalkonferenz der Lehrerverbände (ROSLO) in einem Beitrag in der «Schweizerischen Lehrerzeitung» fest, dass «die Lehrerschaft in allen Kantonen nicht überzeugt werden konnte von der Notwendigkeit einer Vorverlegung des Französischunterrichts auf die Primarstufe. Sie sieht darin keine wesentlichen Vorteile, dafür aber eine Mehrbelastung für Schüler und Lehrer, erhöhten Stoff- und Selektionsdruck, Verstärkung der Kopflastigkeit unseres Schulwesens, eine weitere Einschränkung des Freiraums des Lehrers innerhalb der Stundentafel» («Reformprojekt Französischunterricht. Stellungnahme», 1979, S. 663).

Trotz des Widerstandes der Lehrerschaft empfahl die EDK-Ost 1986 den ihr angeschlossenen Kantonen die Vorverlegung des Französischunterrichts 
spätestens bis zum Jahr 1996. Die Umsetzung konnte im Kanton Zürich auch deshalb in die Wege geleitet werden, weil eine kantonale Volksinitiative, die sich gegen den frühen Beginn des Französischunterrichts richtete, am 25. September 1988 vom Stimmvolk abgelehnt wurde. Die Einführung konnte sich auf jahrelange Erfahrung mit Versuchsklassen stützen, denn zwischen 1967 und 1989 waren im Kanton Zürich mehrere Versuche durchgeführt worden (Regierungsrat des Kantons Zürich, 1992).

Die Ideen, Programme und Empfehlungen des Europarates drangen zwar bis zur kantonalen Ebene, doch erfuhren sie insbesondere bei den Lehrpersonen eine starke Bedeutungsverschiebung, was sich in den Zeitschriften der Lehrpersonen spiegelt. Denn diese sind ebenfalls als Vermittlungsinstanz für kulturelle Transfers zu sehen. Als Diskursorgane übernehmen sie wichtige Vermittlungsfunktionen im Prozess des Transfers (Caruso et al., 2014, S. 42). Die Bedeutungsverschiebung des Transferobjekts Fremdsprachenunterricht zeigt sich darin, dass in den Zeitschriften kaum Bezüge zur «europäischen Idee» zu finden sind. Stattdessen wird der Nutzen des frühen Beginns hinterfragt oder ein Stoffabbau in anderen Fächern befürchtet.

Die Bemühungen sowohl des Europarates als auch der EDK erzeugten auf kantonaler Ebene einerseits Widerstand, insbesondere bei den Lehrpersonen. Andererseits stellten sie die Kantone vor eine grosse organisatorische Aufgabe: Vor der Umsetzung der Einführung musste die Ausbildung der Lehrpersonen angepasst werden bzw. den amtierenden Lehrpersonen Weiterbildungen in Französisch angeboten werden, neue Lehrmittel mussten erschaffen werden, Schulgesetze und Lehrpläne angepasst werden etc. Der Prozess der Umsetzung führte die praktischen Probleme vor Augen. Dies zeigt, dass die Wissensordnung nicht von heute auf Morgen geändert oder angepasst werden kann.

\section{Die Wissensordnung des Fremdsprachen- unterrichts auf dem Prüfstand}

Die Erweiterung des Fremdsprachenunterrichts in der Schweiz wurde über kulturelle Transfers aus dem Europarat angestossen. Mittels verschiedener Vermittlungsinstanzen auf transnationaler, nationaler und kantonaler Ebene wurde das Transferobjekt Fremdsprachenunterricht immer wieder neu interpretiert und erfuhr so eine Bedeutungsverschiebung. In der Legitimation für die Ausweitung des Fremdsprachenunterrichts vermischten sich die Argumentationsebenen. Sowohl die ideelle Zielsetzung des Europarates als auch der EDK nach besserer Kommunikation zwischen den Sprachregionen verlor sich auf der regionalen und kantonalen Ebene. Pragmatische Probleme der Umsetzung dominierten den Diskurs. Die geforderte neue Wissensordnung bezüglich des Fremdsprachenunterrichts durch den Europarat, die EDK und ihre Regionalkonferenzen wurden auf den Prüfstand gestellt: durch die bildungspolitischen Akteure, die Lehrpersonen oder das Volk, wie im Kanton Zürich über die Volksinitiative. 
Wie die anderen Schulfächer ist das Schulfach Fremdsprache ein Produkt von gesellschaftlichen Konstruktionsprozessen. Der Ruf nach einer Reform des fremdsprachlichen Unterrichts kam in der Vergangenheit in regelmässigen Abständen von etwa fünfzig Jahren auf. Die Reformforderungen wurden fast immer fachintern begründet. Der Europarat und die EDK reagierten mit den Empfehlungen auf gesellschaftspolitische Zeitströmungen. Die veränderten gesellschaftlichen Rahmenbedingungen setzten neue Eckwerte für sprachenpolitische als auch schulische Entscheidungen. Der Anstoss für die Reform kam hier allerdings erstmals von aussen (Freudenstein, 1989). Gleichzeitig zeigt das Fremdsprachenfach, dass das schulische Wissen keine blosse Reduktion und Rekonstruktion wissenschaftlichen Wissens ist. Vielmehr fällt auf, dass bei diesem Schulfach nicht auf ihre wissenschaftliche Disziplin rekurriert wird, z.B. beim Französischunterricht auf die Romanistik, sondern auf die Neurophysiologie oder Entwicklungspsychologie (z.B. EDK, 1974b, S. 16f.).

Die Änderung der Wissensordnung war nicht nur in der Schweiz ein langwieriger Prozess, sondern ebenso in anderen Ländern des Europarates (Trim, 2007). In der Schweiz diente das Schulfach als Gefäss für die Thematisierung von verschiedenen Problemen, die sich weniger an den ideellen Zielen orientierten, als an den alltagspraktischen Problemen, die eine Änderung der Wissensordnung nach sich zieht. Die gesellschaftlichen Prozesse der zunehmenden Mobilität (innerhalb und ausserhalb der Schweiz), der zunehmenden Globalisierung und Migration sowie die steigenden Anforderungen der Wirtschaft stiessen die kulturellen Transferprozesse an. Bei der Umsetzung der Empfehlungen waren die Kantone mit organisatorischen Aufgaben konfrontiert wie der Anpassung der Ausbildung der Lehrpersonen, der Stundentafeln oder der Konzipierung neuer Lehrmittel. All diese Anpassungen ziehen lange und komplexe Prozesse nach sich, in die verschiedene Akteure und Akteursgruppen eingebunden sind.

Die Austauschbeziehungen im Bereich des Fremdsprachenunterrichts zwischen den Europaratländern endeten nicht mit den hier erwähnten Resolutionen und der Empfehlung der EDK von 1975. Im Gegenteil, sie intensivierten sich und trieben die Schaffung eines europäischen Sprachencurriculums weiter voran. Bei der Erarbeitung des Gemeinsamen Europäischen Referenzrahmens für Sprachen (GER) beispielsweise war die EDK massgeblich mitbeteiligt. Der Grundstein für den GER wurde 1991 an einem Symposium des Europarats in Rüschlikon ( $\mathrm{ZH})$ gelegt. Die Deskriptoren zur Beschreibung der Sprachenkompetenzen, die später als Grundlage für den GER dienten, wurden in einem Schweizer Forschungsprojekt entwickelt (CE Rat für kulturelle Zusammenarbeit, 2001; Harsch, 2007; Hutterli, 2012). Solche Massnahmen wie der GER zur Standardisierung von Bildungsqualität und ihrer Überprüfung im nationalen und internationalen Vergleich werden in der Regel mit Globalisierungsprozessen in Verbindung gebracht (Thürmann, 2016). Die Umsetzung der Empfehlungen legte den Grundstein für diese spätere Standardisierung und die zunehmenden Globalisierungsprozesse im Bildungsbereich. Gleichzeitig waren 
die Empfehlungen der EDK von 1975 bis dahin ein noch nicht existierendes Novum für die Schweizer Volksschulen, weil die EDK damit erstmals ein nationales Curriculum skizzierte, das den internationalen Anschluss suchte (Gut \& Badertscher, 2002).

Im Bericht zur Vernehmlassung der Empfehlungen postulierte die EDK, dass sich die Schweiz zusammen mit den anderen Nationen am europäischen «Feldzug des fremdsprachlichen Unterrichts» beteiligen solle (EDK, 1974b, S. 11). Fünfzehn Jahre später bekräftigte die EDK diesen Standpunkt:

Die Beschäftigung mit sprachlicher und kultureller Vielfalt wie sie in der Schweiz und in Europa besteht, ist aus staats-, kultur-, bildungs-, gesellschafts- und wirtschaftspolitischen Gründen eine DAUERAUFGABE, die nie aufgegeben werden darf. Die EDK hat den Auftrag, diese Aufgabe zu koordinieren und mitzutragen (EDK, 1989, S. 21, Hervorh. im Original).

Die aktuellen Debatten zum Fremdsprachenunterricht zeigen, dass diese Daueraufgabe die EDK und die Kantone nach wie vor beschäftigt.

\section{Anmerkungen}

1 Die Sprachenstrategie der EDK von 2004 führte zu einer Modifikation ohne Priorisierung einer Landessprache: «Spätestens bis zum 5. Schuljahr setzt der Unterricht von mindestens zwei Fremdsprachen ein, wovon mindestens eine Landessprache. Die erste Fremdsprache beginnt spätestens ab dem 3. Schuljahr, die zweite spätestens ab dem 5. Schuljahr» (EDK, 2004, S. 7).

2 Andere internationale Vereinigungen wie die UNO, die OECD oder die EU verfolgten und verfolgen ebenfalls Programme zur Förderung des Fremdsprachenunterrichts. Der vorliegende Beitrag ist auf die Programme und Bemühungen des Europarates beschränkt, weil die Schweiz seit 1963 ein aktives Mitglied ist und in den Empfehlungen der EDK explizit auf die Länder des Europarates verwiesen wird.

3 Die EDK gibt erst seit 1969 Jahresberichte heraus. Sie vermitteln einen Überblick über die Aktivitäten und Projekte der EDK (Badertscher, 1997a).

4 Das Informationsbulletin der EDK erschien von 1974 bis 1986 . Verschiedene Nummern waren ausschliesslich dem Fremdsprachenunterricht gewidmet.

5 Wiedenkeller bietet einen strukturierten Überblick über kantonale Studien zum Fremdsprachenunterricht (Wiedenkeller, 2013). Das Institut für Mehrsprachigkeit veröffentlicht jährlich eine Jahresbiographie zur Schweizer Mehrsprachigkeitsforschung, die online abrufbar ist (Zugriff am 20.02.2018): http://www.institut-mehrsprachigkeit.ch/de/publications

6 Das Transferkonzept hat seinen Ursprung in den 1980er Jahren am Centre nationale de la recherche scientifique (CNRS). Ursprünglich in den Literatur- und Kulturwissenschaften entwickelt, hat sich der Ansatz in vielen Bereichen der Geschichtswissenschaft etabliert (Espagne \& Werner, 1985, 1987; Middell, 2016).

7 Wegweisend für die Gründung des Europarats war Churchills berühmte Rede in Zürich vom 19. September 1946. Darin entwarf er eine Idee der "Vereinigten Staaten von Europa" (Brummer, 2008). Laut Churchill galt es, "to recreate the European Family, or as much of it as we can, and to provide it with a structure under which it can dwell in peace, in safety and in freedom. We must build a kind of United States of Europe» (Churchill, 1946, S. 1). Den ersten praktischen Schritt zu den «Vereinigten Staaten von Europa» sah Churchill darin, «to form a Council of Europe» (Churchill, 1946, S. 3). 
8 Siehe systematische Rechtssammlung des Bundes zum Internationalen Recht in Bezug auf das Europäische Kulturabkommen, Zugriff am 26.02.2018 unter https://www.admin.ch/ opc/de/classified-compilation/19540245/index.html

9 Neben den Empfehlungen der Erziehungsminister von 1961 und 1962 und der Resolution des Ministerkommitees von 1969, hat auch die Parlamentarische Versammlung 1968 den Beginn des Fremdsprachenunterrichts im Alter von 8 bis 10 Jahren gefordert (CE Assemblée parlementaire, 1968). Die zweite wichtige Empfehlung des Ministerkomitees folgte 1982 (CE Comité des Ministres, 1982). Eine Zusammenstellung weiterer wichtiger Empfehlungen bis 2004 finden sich in Jostes (2004) sowie auf der Website des Europarates. Zugriff am 20.02.2018 unter http://www.coe.int/t/dg4/linguistic/Conventions_ FR.asp

10 Die besondere föderalistische Situation der Schweiz und ihrer Bildungspolitik führte beim Beitritt zum Europäischen Kulturabkommen zu einer Art «Disclaimer», der 1962 unter «Vorbehalte und Erklärungen» formuliert wurde: «Mit Rücksicht auf die föderalistische Struktur der Schweiz und insbesondere die Zuständigkeit der Kantone auf dem Gebiet der Erziehung und der Kultur, wie sie sich aus der Bundesverfassung ergibt, wird diese Zuständigkeit bei der Anwendung des Abkommens durch die Schweiz vorbehalten» (CE, 1954 [2003], S. 6).

11 Die Veränderung der Rolle der EDK hinsichtlich internationaler Beziehungen, fand parallel zu ihrer Ausdifferenzierung als komplexer korporativer Akteur statt, der zahlreiche Policy-Instrumente wie Suborganisationen, ein Generalsekretariat, eine Forschungsstelle und verschiedene Ausschüsse entwickelte.

12 Die 8. Konferenz der europäischen Erziehungsminister fand vom 5. bis 7. Juni 1973 in Bern statt und befasste sich mit der Schulung der 16 bis 19-jährigen Jugendlichen, Zugriff am 09.03.2018 unter http://www.coe.int/fr/web/education-minister-conference/previous-conferences?desktop=true. Auf dieser Website findet sich ebenfalls eine Übersicht zu allen Konferenzen der Erziehungsminister und -ministerinnen seit 1959 und den dort verabschiedeten Resolutionen.

13 Die vier Regionalkonferenzen waren die Regionalkonferenz der Romandie und des Tessins sowie die Regionalkonferenzen der Nordwest-, Ost- und Zentralschweiz.

14 Bis zum Lehrplan von 1995 erfolgte der Übertritt in die Sekundar- oder Primaroberschule im Kanton Bern am Ende des 4. Schuljahres. Mit dem Lehrplan von 1995 wurde die sechsjährige Primarstufe und die dreijährige Sekundarstufe I mit Real- und Sekundarschule eingeführt (Erziehungsdirektion des Kantons Bern, 1995).

15 Aktuell beginnt der Französischunterricht im Kanton Aargau im 6. Schuljahr. Der Beginn im 5. Schuljahr wird mit dem Inkrafttreten des neuen Aargauer Lehrplans (Lehrplan 21) koordiniert. Zugriff am 19.02.2018 unter https://www.ag.ch/de/bks/kindergarten_volksschule/unterricht_schulbetrieb/lehrplan_lehrmittel_volksschule/sprachen/franzoesisch/ franzoesisch.jsp. Die Schülerinnen und Schüler im Kanton Aargau lernen seit dem Schuljahr 2008/2009 ab dem 3. Schuljahr Englisch (Kanton Aargau, 2008).

\section{Literatur}

Bader, U. \& Schaer, U. (2005). Evaluation Englisch in den 6. Klassen Appenzell Innerrhoden 2005. Aarau: Pädagogische Hochschule Fachhochschule Nordwestschweiz.

Badertscher, H. (1997a). Zur Quellenlage der Geschichte der EDK. In H. Badertscher (Hrsg.), Die Schweizerische Konferenz der kantonalen Erziehungsdirektoren 1897 bis 1997. Entstehung, Geschichte, Wirkung (S. 292-294). Bern: Haupt.

Badertscher, H. (1997b). Die EDK als Instrument der Kooperation im Bildungsföderalismus seit 1968. In H. Badertscher (Hrsg.), Die Schweizerische Konferenz der kantonalen Erziehungsdirektoren 1897 bis 1997. Entstehung, Geschichte, Wirkung (S. 173-227). Bern: Haupt. 
Beacco, J.-C. \& Byram, M. (2007). Guide for the Development of Language Education Policies in Europe. From Linguistic Diversity to Plurilingual Education. Main Version. Strasbourg: Council of Europe. Zugriff am 23.01.2018 unter http://www.coe.int/t/dg4/linguistic/ Source/Guide_Main_Beacco2007_EN.doc

Brummer, K. (2008). Der Europarat. Eine Einfuihrung. Wiesbaden: VS.

Bühler, U. (1972). Empirische und lernpsychologische Beiträge zur Wahl des Zeitpunktes für den Fremdsprachunterrichtsbeginn. Lernpsychologisch interpretierte Leistungsmessungen im Französischunterricht an Primarschulen des Kantons Zürich. Zürich: Orell Füssli.

Caruso, M., Koinzer, Th., Mayer, Ch. \& Priem, K. (2014). Einführung. In M. Caruso, Th. Koinzer, Ch. Mayer \& K. Priem (Hrsg.), Zirkulation und Transformation. Pädagogische Grenzüberschreitungen in historischer Perspektive (S. 8-11). Köln: Böhlau.

CE. (1954 [2003]). Europäisches Kulturabkommen. Abgeschlossen in Paris am 19. Dezember 1954. Von der Bundesversammlung genehmigt am 5. Juni 1962. Stand am 23. September 2003, Zugriff am 25.01.2018 unter https://www.admin.ch/opc/de/classified-compilation/19540245/200308270000/0.440.1.pdf

CE. (1969). Résolution (69) 2 (adapté pas les Délégués des Ministres le 25 janvier 1969). Programme intensifié d'enseignement des langues vivantes pour l'Europe. Zugriff am 04.01.2018 unter https://www.coe.int/fr/web/common-european-framework-reference-languages/recommendations

CE. (1974). Symposium sur l'enseignement précoce d'une langue vivante. Strasbourg: Conseil de l'europe. Zugriff am 12.02.2018 unter https://files.eric.ed.gov/fulltext/ED094581.pdf

CE Assemblée parlementaire. (1968). Recommandation 535. Promotion de l'enseignement des langues vivantes en Europe. Zugriff am 04.02.2018 unter http://semantic-pace.net

CE Comité des Ministres. (1982). Recommandation $n^{\circ} R$ (82)18 du Comité des Ministres aux États Membres concernant les langues vivantes (adopté par le Comité des Ministres le 24 septembre 1982, lors de la 350e réunion des Délégués des Ministres). Zugriff am 04.02.2018 unter https://rm.coe.int/16804f6367

CE Rat für kulturelle Zusammenarbeit. (2001). Gemeinsamer europäischer Referenzrahmen für Sprachen: lernen, lehren, beurteilen. Strassburg: Europarat. Zugriff am 26.01.2018 unter https://www2.ac-lyon.fr/enseigne/allemand/Referenzrahmen2001.pdf

Christ, H. (2003). Sprachenpolitik und das Lehren und Lernen fremder Sprachen. In K.-R. Bausch, H. Christ \& H.-J. Krumm (Hrsg.), Handbuch Fremdsprachenunterricht (S. 102-110). Tübingen: Francke.

Christ, I. (2003). Staatliche Regelungen für den Fremdsprachenunterricht: Curricula, Richtlinien, Lehrpläne. In K.-R. Bausch, H. Christ \& H.-J. Krumm (Hrsg.), Handbuch Fremdsprachenunterricht (S. 71-76). Tübingen: Francke.

Christ, H. \& de Cillia, R. (2003). Geschichte des Fremdsprachenunterrichts in deutschsprachigen Ländern seit 1945. In K.-R. Bausch, H. Christ \& H.-J. Krumm (Hrsg.), Handbuch Fremdsprachenunterricht (S. 614-621). Tübingen: Francke.

Churchill, W. (1946). Universität Zürich, 19. September 1946. Zugriff am 26.01.2018 unter http://www.churchill-in-zurich.ch/site/assets/files/1807/rede_winston_churchill_englisch.pdf

Die Tagungen der Erziehungsdirektorenkonferenz 1963. (1964). Archiv für das schweizerische Unterrichtswesen, 49, 108-113.

Die Vorverlegung des Französischunterrichts in der Ostschweiz (1986). Schweizer Schule, 73, (1), 26.

EDK (1972). Jahresbericht 1971/1972. Zug/Genf, Oktober 1972. Zugriff am 14.01.2018 unter https://edudoc.ch/record/24488/files/1971-1972_d.pdf

EDK (1973). Jahresbericht 1972/1973. Zug/Genf, Oktober 1973. Zugriff am 14.01.2018 unter https://edudoc.ch/record/24490/files/1972-1973_d.pdf

EDK (1974a). Expertenkommission zur Einführung und Koordination des Fremdsprachunterrichts in der obligatorischen Schulzeit (Informationsbulletin, 1). Genf: Wissenschaftliches Sekretariat der Expertenkommission. 
EDK (1974b). Bericht und Anträge zur Einführung und Koordination des Fremdsprachunterrichts in der obligatorischen Schulzeit (Informationsbulletin, 2a). Genf: Wissenschaftliches Sekretariat der Expertenkommission.

EDK (1974c). Jahresbericht 1973/1974. Zürich/Genf, Oktober 1974. Zugriff am 14.01.2018 unter https://edudoc.ch/record/24492/files/1973-1974_d.pdf

EDK (1975). Empfehlungen und Beschlüsse betreffend Einführung, Reform und Koordination des Unterrichts in der zweiten Landessprache für alle Schüler während der obligatorischen Schulzeit. vom 30. Oktober 1975. In EDK. (1995), Empfehlungen und Beschlüsse (S. 23-37). Bern: EDK. Zugriff am 26.01.2018 unter http://edudoc.ch/record/24416/files/D36A.pdf

EDK (1989). Das Projekt Fremdsprachunterricht / Langue 2 am Ende der achtziger Jahre. Geschichtlicher Rückblick - Beurteilung der Lage - Optionen für die Zukunft. Bern: EDK. Zugriff am 27.01.2018 unter http://edudoc.ch/record/114114/files/1989.pdf

EDK (2004). Sprachenunterricht in der obligatorischen Schule: Strategie der EDK und Arbeitsplan für die Gesamtschweizerische Koordination. Bern: EDK. Zugriff am 26.02.2018 unter http://edudoc.ch/record/30008/files/Sprachen_d.pdf

Egger, E. \& Blanc, E. (1977). Neuerungen im schweizerischen Schulwesen. Besonderheiten und Tendenzen (Informationsbulletin, 9). Genf: Schweizerische Dokumentationsstelle für Schul- und Bildungsfragen.

Erziehungsdepartement des Kantons Aargau. (1983). Lehrplankonzept für die Volksschule. Fassung für die Vernehmlassung. Aarau: Erziehungsdepartement des Kantons Aargau.

Erziehungsdirektion des Kantons Bern (1966). Lehrplan für die Primarschulen des Kantons Bern. Bern: Staatlicher Lehrmittelverlag.

Erziehungsdirektion des Kantons Bern (1983). Lehrplan für die Primar- und Sekundarschulen des Kantons Bern. Ausgabe Primarschulen. Bern: Staatlicher Lehrmittelverlag.

Erziehungsdirektion des Kantons Bern (1995). Lehrplan Volksschule. Primarstufe und Sekundarstufe I. Bern: Staatlicher Lehrmittelverlag.

Erziehungsdirektion des Kantons Zürich (1993). Lehrplan für die Volksschule des Kantons Zürich. Zürich: Lehrmittelverlag des Kantons Zürich.

Espagne, M. \& Werner, M. (1985). Deutsch-französischer Kulturtransfer im 18. und 19. Jahrhundert. $\mathrm{Zu}$ einem neuen interdisziplinären Forschungsprogramm des C.N.R.S. Francia. Forschungen zur westeuropäischen Geschichte 13, 502-520. Zugriff am 29.01.2018 unter http://www.perspectivia.net/publikationen/francia/

Espagne, M. \& Werner, M. (1987). La construction d'une référence culturelle allemande en France. Genèse et histoire (1750-1914). Ahess, 42, (4), 969-992. Zugriff am 29.10.2016 unter http://www.persee.fr/doc/ahess_0395-2649_1987_num_42_4_283428

Extermann, B. (2013). Une langue étrangère et nationale. Histoire de l'enseignement de l'allemand en Suisse romande (1790-1940). Neuchâtel: Alphil.

Fontaine, A. (2015). Aux heures suisses de l'école républicaine: un siècle de transferts culturels et de déclinaisons pédagogiques dans l'espace franco-romand. Paris: Demopolis.

Freudenstein, R. (1989). Fremdsprachen in der Schule nach 1992. Politik und Sprachenlernen auf dem Weg zur europäischen Integration. In E. Kleinschmidt (Hrsg.), Fremdsprachenunterricht zwischen Sprachenpolitik und Praxis. Festschrift für Herbert Christ zum 60. Geburtstag (S. 14-20). Tübingen: G. Narr.

Gesetz über die Sekundarschulen vom 12. März 1839 (1839). In Gesetze, Dekrete und Verordnungen der Republik Bern. Neunter Band (S. 61-69). Zugriff am 02.02.2018 unter https:// www.e-periodica.ch/digbib/view?pid=gdv-001:1839:9\#4

Giudici, A. \& Grizelj, S. (2014). Vom Berufs- und Elitewissen zum Garant des nationalen Zusammenhalts. Die Fremdsprachen in den Lehrplänen der Schweizer Volksschulen seit 1830. Babylonia, 74, (3), 60-65.

Giudici, A. \& Grizelj, S. (2017). National unity in cultural diversity: how national and linguistic identities affected Swiss language curricula (1914-1961). Paedagogica Historica, 53, (1-2), 137-154. 
Goodson, I. (1997). The changing curriculum. Studies in social construction. New York, NY: Lang.

Goodson, I. (1999). Entstehung eines Schulfaches. In I. Goodson, S. Hopmann \& K. Riquarts (Hrsg.), Das Schulfach als Handlungsrahmen. Vergleichende Untersuchung zur Geschichte und Funktion der Schulfächer (S. 151-176). Köln: Böhlau.

Gut, K. \& Badertscher, H. (2002). Formen und Funktionen des Unterrichts moderner Fremdsprachen in der Schweiz des 20. Jahrhunderts. In E. Lechner (Hrsg.), Formen und Funktionen des Fremdsprachenunterrichts im Europa des 20. Jahrhunderts (S. 157-188). Frankfurt am Main: Lang.

Haas W. (2010). Do you speak Swiss? Sprachenvielfalt und Sprachkompetenz in der Schweiz. Nationales Forschungsprogramm NFP 56. Zürich: Verlag Neue Zürcher Zeitung.

Haenni Hoti, A., Müller, M., Heinzmann, S., Wicki, W. \& Werlen, E. (2009). Frühenglisch - Überforderung oder Chance? Eine Längsschnittstudie zur Wirksamkeit des Fremdsprachenunterrichts auf der Primarstufe (Forschungsbericht Nr. 20 der Pädagogischen Hochschule Zentralschweiz). Luzern: Hochschule Luzern. Zugriff am 20.02.2018 unter https:// edudoc.ch/record/32473/files/phzlu_fe_Schlussbericht_NFP56.pdf

Harsch, C. (2007). Der gemeinsame europäische Referenzrahmen für Sprachen. Leistung und Grenzen. Saarbrücken: VDM Verlag Dr. Müller.

Husfeldt, V. \& Bader Lehmann, U. (2009). Englisch an der Primarschule. Lernstandserhebung im Kanton Aargau. Aarau: BKS. Zugriff am 18.02.2018 unter https://edudoc.ch/ record/35204/files/zu09067.pdf

Hutterli, S. (Hrsg.) (2012). Koordination des Sprachenunterrichts in der Schweiz. Aktueller Stand, Entwicklungen, Ausblick. Bern: EDK.

Innerschweizer Erziehungsdirektorenkonferenz IEDK. (1985). Schulkoordination und Schulreform in der Zentralschweiz. Festschrift zum zwanzigjährigen Bestehen der Innerschweiz Erziehungsdirektorenkonferenz. Luzern: Zentralschweizerischer Beratungsdienst für Schulfragen.

Jostes, B. (2004). Die Sprachenpolitik des Europarats: Nähe und Distanz in der europäischen Mehrsprachigkeit. Grenzgänge, 11, (22), 6-30.

Kanton Aargau: Kontroverse um Französischunterricht (1977). Schweizer Schule, 64, (14-15), 482-483.

Kanton Aargau. (2008). Informationsbroschüre für Eltern. Aarau: Departement Bildung, Kultur und Sport. Zugriff am 10.01.2018 unter http://www.sprachenunterricht.ch/sites/ default/files/Elternhandoutenglisch_0.pdf

Krumm, H.-J. (2016a). Sprachenpolitik und das Lernen und Lehren von Sprachen. In E. Burwitz-Melzer, G. Mehlhorn, C. Riemer, K.-R. Bausch \& H.-J. Krumm (Hrsg.), Handbuch Fremdsprachenunterricht (S. 45-51). Tübingen: A. Francke Verlag.

Krumm, H.-J. (2016b). Förderung des Lernens und Lehrens von Sprachen durch Europarat und Europäische Union. In E. Burwitz-Melzer, G. Mehlhorn, C. Riemer, K.-R. Bausch \& H.-J. Krumm (Hrsg.), Handbuch Fremdsprachenunterricht (S. 633-637). Tübingen: A. Francke Verlag.

Lüdi, G., Pekarek, S. \& Saudan, V. (2001). Französischlernen in der Deutschschweiz. Zur Entwicklung der diskursiven Fähigkeiten innerhalb und ausserhalb der Schule. Chur: Rüegger.

Manz, K. (2011). "Schulkoordination ja - aber nicht so!». Die Anfänge der schweizerischen Schulkoordination (1960-1985). Bern: hep.

Middell, M. (2016). Kulturtransfer, Transferts culturels (Version: 1.0). In Docupedia-Zeitgeschichte (Stand 28.01.2016). Zugriff am 29.01.2018 unter https://docupedia.de/zg/ Kulturtransfer

Namer, G. (1981). Die dreifache Legitimation. Ein Modell für eine Soziologie des Wissens. In N. Stehr \& M. Volker (Hrsg.), Wissenssoziologie (S. 191-205). Opladen: Westdeutscher Verlag.

Peyer, E., Andexlinger, M., Kofler, K. \& Lenz, P. (2016). Projekt Fremdsprachenevaluation $B K Z$. (Schlussbericht zu den Sprachkompetenztests). Fribourg: Institut für Mehrspra- 
chigkeit. Zugriff am 29.01.2018 unter http://doc.rero.ch/record/259287/files/Bericht_ BKZ_Fremdsprachenevaluation_2016-01-26.pdf

Rechenschaftsbericht des Regierungsrates an den Kantonsrat (1980). Schwyz: Regierungsrat. Rechenschaftsbericht des Regierungsrates an den Kantonsrat (1988). Schwyz: Regierungsrat. Rechenschaftsbericht des Regierungsrates an den Kantonsrat (1989). Schwyz: Regierungsrat. Rechenschaftsbericht des Regierungsrates an den Kantonsrat (1990). Schwyz: Regierungsrat. Rechenschaftsbericht des Regierungsrates an den Kantonsrat (1993). Schwyz: Regierungsrat. Regierungsrat des Kantons Zürich. (1992). Auszug aus dem Protokoll des Regierungsrates des Kantons Zürich. Sitzung vom 20. Mai 1992. 1513. Interpellation. Zugriff am 27.02.2018 unter https://www.kantonsrat.zh.ch/geschaefte/geschaefte.aspx

Reformprojekt Französischunterricht. Stellungnahme (1979). Schweizerische Lehrerzeitung, 124, (17), 663.

Sarter, H. (2013). Mehrsprachigkeit und Schule. Eine Einführung. Darmstadt: WBG.

Schwob, I. \& Hexel, D. (2008). ALLEVAL. L'enseignement de l'allemand à l'école obligatoire à Genève. Ressources, processus, résultats. Genève: SRED. Zugriff am 20.02.2018 unter https://www.ge.ch/recherche-education/doc/publications/docsred/2008/alleval.pdf

Staatsarchiv Luzern, EDK (1963). Akten und Druckschriften 1895-2000, A 1270/1530. In Moderne Sprachen - spezifische und interregionale Strukturanalyse des Unterrichts moderner Sprachen, 1963-1976. «Etudes sur la structure et l'envergure de l'enseignement des langues vivantes en Europe par M. Neumeister.».

Stauffacher, M. (2016). 50 Jahre EDK-Ost. 1966-2016. St. Gallen: Regionalsekretariat EDK-Ost. Zugriff am 31.01.2018 unter http://edk-ost.d-edk.ch/

Stauffer, M. (1999). Aspekte des Gesamtsprachenkonzepts: Ergebnisse einer IDES-Umfrage in der Schweiz und im Fürstentum Liechtenstein. Bern: EDK/IDES. Zugriff am 02.01.2018 https://edudoc.ch/record/32242?ln=de

Stillhart, Th. (2005). Regionale Bildungspolitik zwischen Autonomie und Kooperation. Festschrift zum 40-jährigen Bestehen der Nordwestschweizerischen Erziehungsdirektorenkonferenz (NW $E D K)$. Bern: Schulverlag blmv.

Stoks, G. (1994). «Sprachen Lernen und der Europäische Bürger» - das aktuelle Fremdsprachenprojekt des Europarates. In V. Zukunftsforum (Hrsg.), Sprachen Lernen - Menschen Verstehen (S. 334-345). Wien: Bundesministerium für Unterricht und Kunst.

Thürmann, E. (2016). Globalisierung und Standardisierung in ihrer Auswirkung auf das Lernen und Lehren von Sprachen. In E. Burwitz-Melzer, G. Mehlhorn, C. Riemer, K.-R. Bausch \& H.-J. Krumm (Hrsg.), Handbuch Fremdsprachenunterricht (S. 51-56). Tübingen: A. Francke Verlag.

Trim, J. L. M. (2007). Les langues vivantes au conseil de l'Europe 1954-1997. La coopération internationale en faveur de l'apprentissage tout au long de la vie, pour une communication efficace, un enrichissement culturel mutuel et la citoyenneté démocratique en Europe. Strasbourg: Division des Politiques linguistiques. Zugriff am 01.02.2018 unter https://www. coe.int/t/dg4/linguistic/Source/TRIM_21janv2007_FR.doc

Wiedenkeller, E. (2013). Strukturierter Überblick über kantonale Studien zum Fremdsprachenunterricht. Analytische Darstellung, inhaltliche und methodische Auswertung. Im Auftrag $\operatorname{der} E D K$. Freiburg: Institut für Mehrsprachigkeit. Zugriff am 30.01.2018 unter https:// edudoc.ch/record/112670/files/2-REF_E2_strukturierter_Ueberblick_d.pdf

Wyder, R. (1984). Die Schweiz und der Europarat 1949-1971: Annäherung und zehn Jahre Mitarbeit in der Parlamentarischen Versammlung (Schriftenreihe der Schweizerischen Gesellschaft für Aussenpolitik, Bd. 10). Bern: Haupt.

Schlagworte: Fremdsprachenunterricht, Landessprachen, Sprachen-Policy, Schweiz, Europarat 


\section{Configuration européenne des savoirs et enseignement des langues étrangères en Suisse (1961-1990)}

\section{Ré sumé}

Le Conseil de l'Europe est depuis sa création en 1949 un acteur important dans le domaine de la politique des langues. Au moyen de résolutions et de recommandations, il définit des objectifs curriculaires pour l'enseignement scolaire des langues étrangères qui n'ont pas de valeur contraignante pour les États membres, mais qui ont entraîné toutefois un processus de reconfiguration des savoirs scolaires, par le biais de transferts culturels. Ainsi, la CDIP s'est emparée des recommandations du Conseil de l'Europe et a publié en 1975 des lignes directrices à l'échelle de la Confédération. Ces lignes directrices visaient tant à promouvoir les langues nationales qu'à aligner la Suisse sur la politique culturelle européenne. Toutefois, au niveau des cantons, la reconfiguration prévue a été retardée pour des raisons pragmatiques et organisationnelles.

Mot-clés: Enseignement des langues étrangères, langues nationales, politique des langues, Suisse, Conseil de l'Europe

\section{Organizzazione europea del sapere e insegnamento delle lingue straniere in Svizzera (1961-1990)}

\section{Riassunto}

Sin dalla sua fondazione nel 1949 il Consiglio d'Europa è un attore importante nell'ambito della politica dell'insegnamento delle lingue. Mediante risoluzioni e raccomandazioni, egli ha contribuito a fissare degli obiettivi dell'insegnamento delle lingue straniere in ambito scolastico. Pur non essendo vincolanti per gli Stati membri, tali obiettivi hanno provocato una trasformazione del sapere scolastico attraverso processi di transfer culturale. In Svizzera, le raccomandazioni del Consiglio d'Europa furono recepite dalla CDPE la quale formulò nel 1975 un curricolo nazionale per le lingue straniere. Nelle intenzioni della CDPE tale curricolo avrebbe dovuto promuovere l'apprendimento delle lingue nazionali e la messa in pratica della politica culturale europea. A livello cantonale, tale riorganizzazione venne tuttavia attuata con un certo ritardo, dovuto a ragioni pragmatiche e organizzative.

Parole chiave: Insegnamento delle lingue straniere, lingue nazionali, politica linguistica, Svizzera, Consiglio d'Europa 


\section{European Order of Knowledge in Swiss Language Teaching (1961-1990)}

\section{Summary}

Since its foundation in 1949, the Council of Europe has been an important player in language education policy. Passing several resolutions and recommendations, it set programmatic goals for language teaching in schools, which were not mandatory for its member states, but still initiated a reorganization of school knowledge through cultural transfers. The Swiss Conference of Cantonal Ministers of Education (EDK) also took up these recommendations when defining a national curriculum for foreign languages in 1975. The aim of the curriculum was to foster the Swiss national languages and implement a European cultural policy. However, within the cantons, the implementation of this reorganisation of knowledge was delayed because of pragmatic and organizational difficulties.

Keywords: Foreign language teaching, national languages, language policy, Switzerland, Council of Europe 
